\title{
Getting a grip on guidelines: occupational contact dermatitis and urticaria
}

Evidence-based guideline development can be seen as one of the major recent developments in efforts to improve patient care, providing clinicians and patients with information on how to manage health problems appropriately in day-to-day practice. ${ }^{1}$ Active involvement of patients and the public in the research process is expected to lead to research which reflects their needs and views, and subsequently is likely to produce results that can be used to improve practice in health and social care. ${ }^{2}$

The British Occupational Health Research Foundation (BOHRF) is a charity which raises and deploys funds for occupational health research of practical value to reduce the enormous cost to employers and workers of work-related illnesses. In March 2010, the BOHRF launched evidence-based guidelines for the prevention, identification and management of occupational contact dermatitis and urticaria. $^{3}$ The guideline development group comprised dermatologists, a GP, occupational physicians, occupational health nurses, safety professionals, employers, and trade union and patient representatives. Previously, the NHS Plus Occupational Health Clinical Effectiveness Unit at the Royal College of Physicians released evidence-based guidelines about latex allergy ${ }^{4}$ and dermatitis, ${ }^{5}$ particularly as they affected healthcare workers in 2008 and 2009 respectively.

GPs face pressure to provide a mounting range of services and targets on behalf of their NHS patients. A total of 28.9 million people are employed in the $\mathrm{UK},{ }^{6}$ meaning that approximately half of the average GP list includes those in active work. However, only one in seven workers can access occupational health provided through their employer. ${ }^{7}$ This unmet need of occupational health provision may well fall to GPs to recognise and act on work-related health problems despite the fact that most GPs have no formal occupational health training.
From April 2010 the medical certificate used for statutory sick pay purposes in the UK was replaced by a version that encourages GPs to certify if a patient is fit for some work; the previous version only allowed GPs to advise whether a patient should or should not refrain from work. In this situation evidence-based guidelines that summarise the evidence relating to the effects of continued, or reduced, exposure to the causes of diseases in the workplace will be helpful to GPs in advising patients and their employers.

\section{DERMATITIS AND URTICARIA}

Working people frequently experience occupational contact dermatitis. Occupational irritant contact dermatitis occurs where physical or chemical agents have a direct damaging effect on the skin; for example, wet work, detergents, alkalis, solvents, and friction. It is the most common type of occupational contact dermatitis and most frequently affects the hands.

Occupational allergic contact dermatitis is a delayed or type IV hypersensitivity reaction as the result of a T-cell mediated immune response to a specific skin sensitizer, such as epoxy resin, nickel, chromium, or colophony. It follows sensitisation to an agent. Further exposure to the specific agent then produces the rash after a delayed period.

Occupational contact urticaria can be non-allergic or allergic, the latter involving an immediate or type I hypersensitivity reaction, associated with the presence of specific immunoglobulin E. It is often associated with proteins in food and latex gloves.

Occupational contact dermatitis may present at any stage in a worker's career. ${ }^{3}$ Workers at highest risk of occupational contact dermatitis and urticaria include those in agriculture, beauticians, chemical workers, cleaners, construction workers, cooks and caterers, electronics workers, farmers, hairdressers, health and social care workers, machine operators, mechanics, and metalworkers. ${ }^{3}$ It is important for GPs to ask patients who present with dermatitis or urticaria about their job and what their work entails, and to be aware of the types of workers most at risk.

Further reasons to suspect an occupational cause of a rash include the location of the rash (occupational dermatoses affect the hands most commonly, followed by the arms and face $^{3}$ ) and the temporal relationship between symptoms and work. ${ }^{3}$ However, neither is a pathognomonic sign of an occupational cause; a temporal relationship may indicate that work has aggravated a pre-existing dermatitis and has not necessarily contributed to, or caused, occupational contact dermatitis.

Exposure to agents at work presents the greatest risk for the development of occupational dermatoses. Some people may be at increased risk through personal risk factors such as atopy, but have poor predictive value and are not reasons to exclude someone from work. Atopy in particular has been examined as a personal risk factor for the development of occupational contact dermatitis but the evidence for any link is conflicting. However, atopic dermatitis particularly in adulthood appears to be an independent risk factor for occupational contact dermatitis. ${ }^{3}$ There is more consistency in studies for a link between atopy in general as a risk factor for occupational contact urticaria, at least in the case of latex. ${ }^{3}$

When seeing a patient, it is necessary to distinguish between non-occupational eczema and occupational contact dermatitis. If an occupational cause is suspected, it is essential to distinguish between irritant and allergic contact dermatitis as management and advice will differ.

Occupational contact dermatitis and urticaria are diagnosed by careful history taking, clinical examination, and patch testing in the case of dermatitis, and skin prick tests or blood tests for specific 
immunoglobulin $\mathrm{E}$ in the case of urticaria. Such tests are best performed in specialist units that can access or prepare suitable test material. Specialist referral should include the patient's occupational history, any temporal relationship between the rash and the patient's work, and hazardous exposures from work. Negative patch testing may indicate irritant contact dermatitis if the history clearly shows a temporal relationship with the rash.

There is little information about the prognosis of contact urticaria. For occupational contact dermatitis, studies suggest a significant proportion of workers continue to experience symptoms and impairment years after diagnosis despite treatment or job change. ${ }^{3}$ There is a wide prognostic outcome between job retention where reasonable control of symptoms in certain occupational settings is possible, and protracted sickness absence and job loss. Most patients continue working in some capacity, albeit sometimes in altered employment. ${ }^{3}$

GPs may find themselves as the only source of medical advice for management of their patients once a diagnosis of occupational contact dermatosis has been established. The GP may assist, not only in providing appropriate medical management to their patient, but, with their patient's consent, by advising the employer about the health problem and its suspected cause. It is then incumbent on the employer to make reasonable adjustments to the work or environment and to seek expert advice should this be necessary.

Further advice and support for employees, GPs, and employers may be accessed through the Employment Medical Advisory Service, which has a right to visit a workplace.

\section{lan King,}

GP and Consultant Occupational Physician, Holmhurst Medical Centre, Redhill, Surrey.

\section{Paul Nicholson,}

Consultant Occupational Physician, Procter \& Gamble, Occupational Health, Egham.

\section{Provenance}

Freely submitted; not peer reviewed.

\section{REFERENCES}

1. Grol R, van Weel C. Getting a grip on guidelines: how to make them more relevant for practice. Br J Gen Pract 2009; 59(562): e143-144.

2. Oliver S. Participatory and evidence-informed general practice. Br J Gen Pract 2009; 59(563): 397-398.

3. Nicholson PJ, Llewellyn D, editors. Occupational contact dermatitis and urticaria. London: British Occupational Health Research Foundation, 2010

http://www.bohrf.org.uk/downloads/OccupationalCont actDermatitisEvidenceReview-Mar2010.pdf (accessed 12 May 2010)

4. NHS Plus, Royal College of Physicians, Faculty of Occupational Medicine. Latex allergy: occupational health aspects of management: a national guideline. London: Royal College of Physicians, 2008.

5. NHS Plus, Royal College of Physicians, Faculty of Occupational Medicine. Dermatitis: occupational aspects of management. A national guideline. London: Royal College of Physicians, 2009.

6. Office for National Statistics. Labour market statistics January 2010.

http://www.statistics.gov.uk/pdfdir/lmsuk0110.pdf (accessed 12 May 2010).

7. Nicholson PJ. Occupational health services in the UK — challenges and opportunities. Occup Med 2004; 54(3): 147-152.

DOI: 10.3399/bjgp10X502074

ADDRESS FOR CORRESPONDENCE

lan King

Mayday University Hospital, Occupational Health, 530 London Road, Croydon, CR7 7YE.

E-mail: ianking@blueyonder.co.uk 